\title{
Submitted: The definitive role of CEUS in an ambiguous case Accepted: of renal cell carcinoma
} 16.07.2021

Published: 16.08.2021

\section{Keywords \\ angiomyolipoma, renal cell carcinoma, ultrasound, contrast-enhanced ultrasound}

\author{
Katarzyna Drelich¹, Monika Zbroja', Weronika Cyranka', \\ Olga Pustelniak¹, Ewa Kopyto¹, Maryla Kuczyńska²
}

${ }^{1}$ Students' Scientific Society at the Department of Interventional Radiology and Neuroradiology, Medical University of Lublin, Lublin, Poland

${ }^{2}$ Department of Interventional Radiology and Neuroradiology, Medical University of Lublin, Lublin, Poland

Correspondence: Katarzyna Drelich, Department of Interventional Radiology and Neuroradiology, Medical University of Lublin, ul. Jaczewskiego 8 20-095 Lublin, Poland; tel.: +48817244154, e-mail:k.drelich1111@gmail.com

DOI: $10.15557 /$ JoU.2021.0040

\begin{abstract}
Angiomyolipoma is a benign tumor consisting of abnormal vessels, smooth muscles, and fatty tissue. Renal cell carcinoma is an insidious neoplasm accounting for approximately $2 \%$ of global cancer diagnoses. Due to similar diagnostic features, the differentiation between the two types is sometimes difficult. We hereby present the case of a 60-year-old patient with no clinical symptoms and a focal lesion in the parenchymal layer of the left kidney incidentally detected on ultrasound examination. The putative diagnosis was angiomyolipoma, which was then confirmed by another ultrasound and computed tomography examinations. However, a further radiologic consultation revealed another probable diagnosis - renal cell carcinoma. Contrast-enhanced ultrasound was conducted, and the enhancement pattern was suggestive of cancer. To sum up, a thorough imaging examination plays an important role in the diagnostic work-up of neoplastic lesions in the kidney. Even then, however, the radiological image of the lesion may be misleading, so differential diagnosis is important for making a proper diagnosis.
\end{abstract}

\section{Introduction}

Angiomyolipoma (AML) is a benign tumor consisting of abnormal vessels, smooth muscles, and fatty tissue. It accounts for $0.3-3 \%$ of all renal masses and is the most common type of benign kidney tumors ${ }^{(1)}$. About $80 \%$ of AMLs present as isolated entities ${ }^{(2)}$, but they are also frequently associated with tuberous sclerosis complex (TSC) (3). AML is radiologically classified into three subtypes according to the amount of fat which is quantified by CT or MRI: fat-rich AML, fat-poor AML, and fat-invisible $\mathrm{AML}^{(4)}$. Abundant fat tissue is a characteristic feature on imaging. However, sometimes there is too little of it to be detected. This poses a difficulty in differentiating AML from renal cell carcinoma (RCC). RCC is an insidious neoplasm accounting for approximately $2 \%$ of global cancer diagno$\operatorname{ses}^{(5)}$. It is classified into three major histological subtypes: clear cell, papillary, and chromophobe ${ }^{(6)}$.
Imaging plays a central role in the diagnosis and management of renal AMLs and RCC. Ultrasound is the examination through which kidney lesions are most commonly diagnosed. Contrast-enhanced ultrasound (CEUS) allows for the identification of active bleeding and is a valuable real-time diagnostic test in abdominal emergencies due to AML. It can also be used for distinguishing between malignant and benign renal lesions.

\section{Case description}

We present the case of a 60 -year-old patient with no clinical symptoms and a focal lesion in the parenchymal layer of the left kidney which was incidentally detected on ultrasound examination. The lesion was well-demarcated, hyperechoic, measuring $22 \times 18 \mathrm{~mm}$, with no blood flow signals in the Doppler option, and located on the dorsolateral side, extending slightly beyond the contour of the kidney. Based on the 


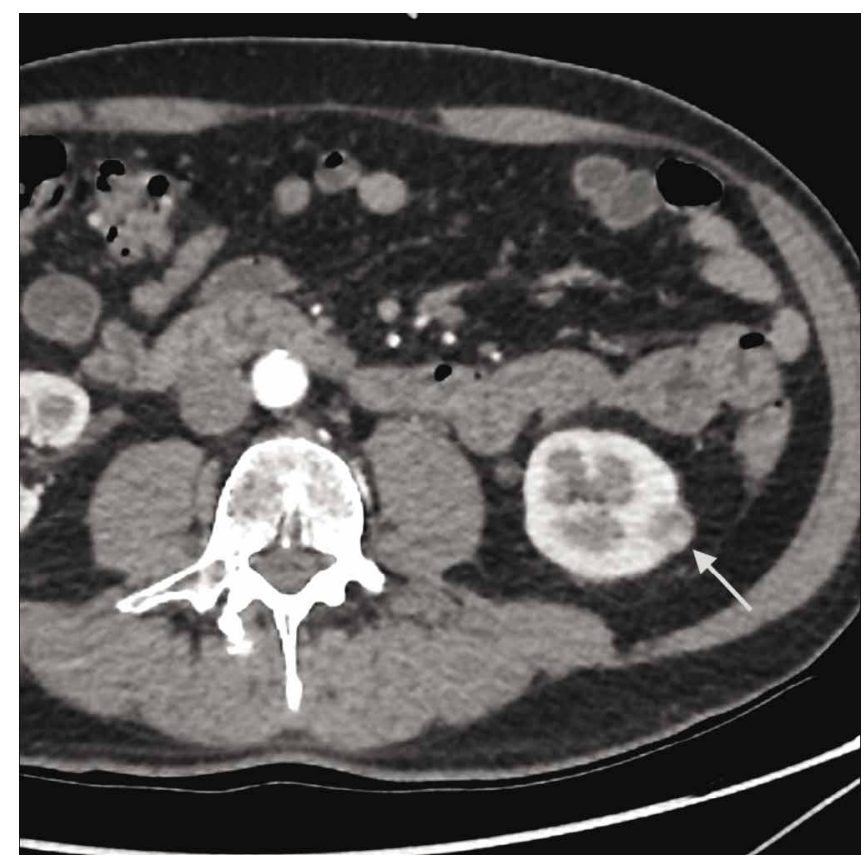

Fig. 1. Well-demarcated lesion in the dorsal aspect of the left kidney; strong, rather peripheral contrast enhancement in the early arterial phase

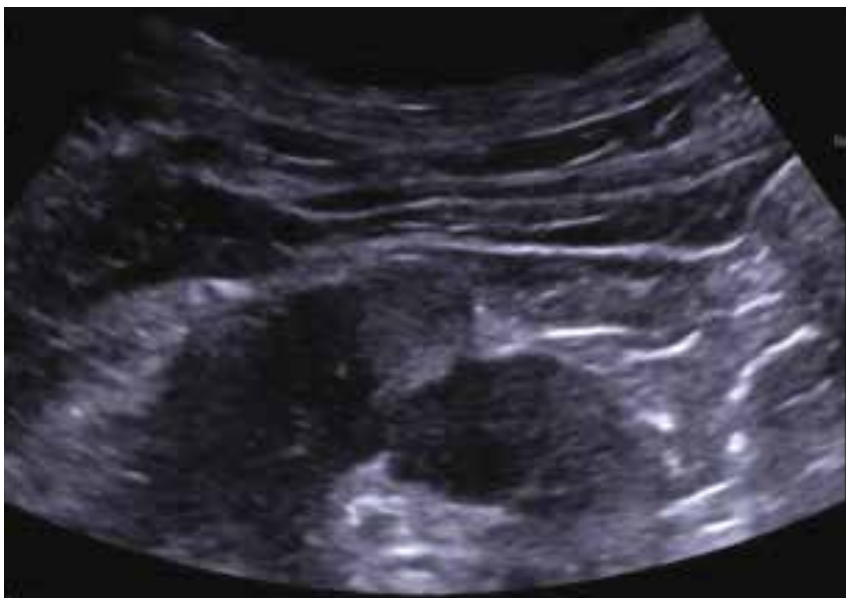

Fig. 3. B-mode image of the well-demarcated, heterogeneously hyperechoic focal lesion within the renal cortex

US findings, the diagnosis of AML was made. In four follow-up ultrasound examinations performed by independent ultrasonographers over a period of 18 months, the morphological image and dimensions of the lesion were invariable, so the diagnosis of AML was maintained.

In order to verify the nature of the lesion (despite the absence of clinical symptoms) CT was performed, revealing an isodense well-demarked lesion in the native phase, with strong enhancement after contrast administration (Visipaque $120 \mathrm{ml}$ ). In the axial projection, there was an area of fatty tissue at the periphery of the lesion, recognized as its component, supporting the diagnosis of AML. Afterwards, however, the patient reported to a highly specialized medical

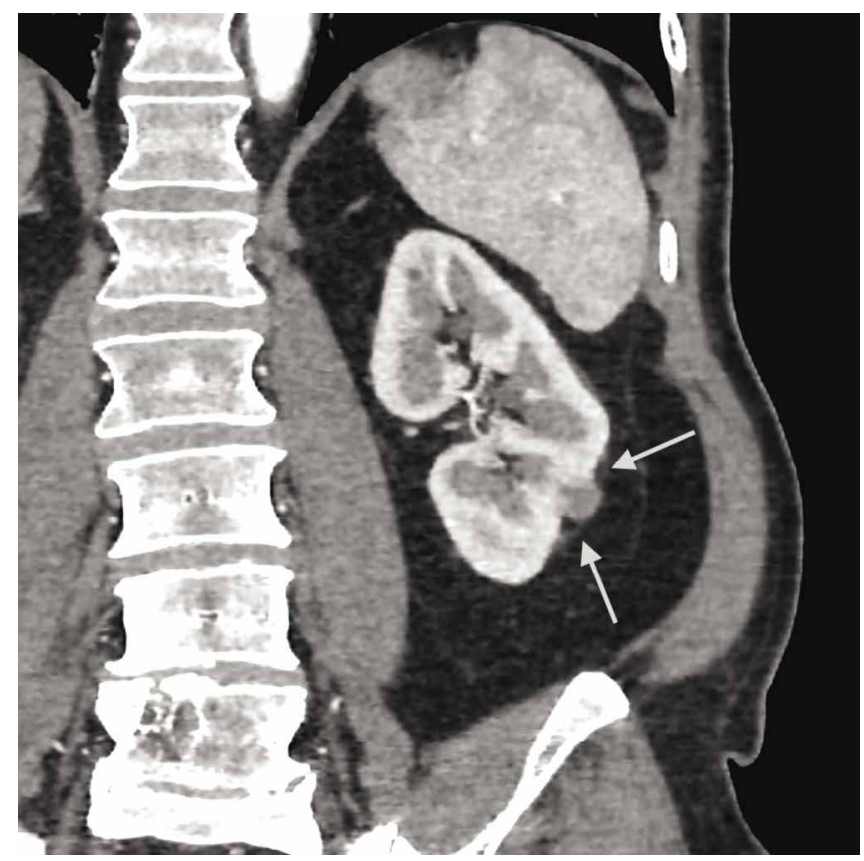

Fig. 2. Renal capsule distorted by the focal mass; coronal CT reconstruction clearly indicated extra-tumor, subcapsular location of the fatty tissue described initially as a component of $A M L$

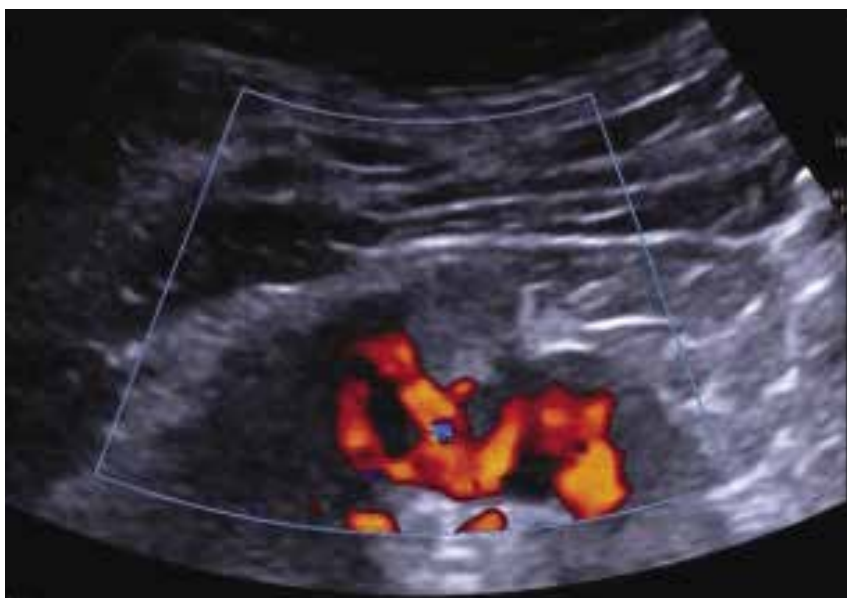

Fig. 4. No apparent flow signals consistent with vascularization of the lesion; prominent modeling of the renal vessels at the tumor margin visible in color Doppler images

center, where his CT images were re-assessed by an independent radiologist who raised the suspicion of the malignant character of the lesion, possibly RCC. Coronal reconstruction of the CT showed that the renal capsule surrounding the tumor was distorted, enabling the subcapsular fatty tissue to protrude around the mass, thus mimicking the fatty component of angiomyolipoma (Fig. 1 and Fig. 2). CEUS examination was then performed three times with $1.2 \mathrm{ml}$ of SonoVue contrast agent (total of $3.6 \mathrm{ml}$ ) at 10 min intervals, each time after complete washout of contrast. Rapid, robust contrast enhancement of the lesion was observed in the early arterial phase, followed by an earlier wash-out as compared to the surrounding renal parenchyma. Although the morphological US image did not allow for unambiguous discrimination 


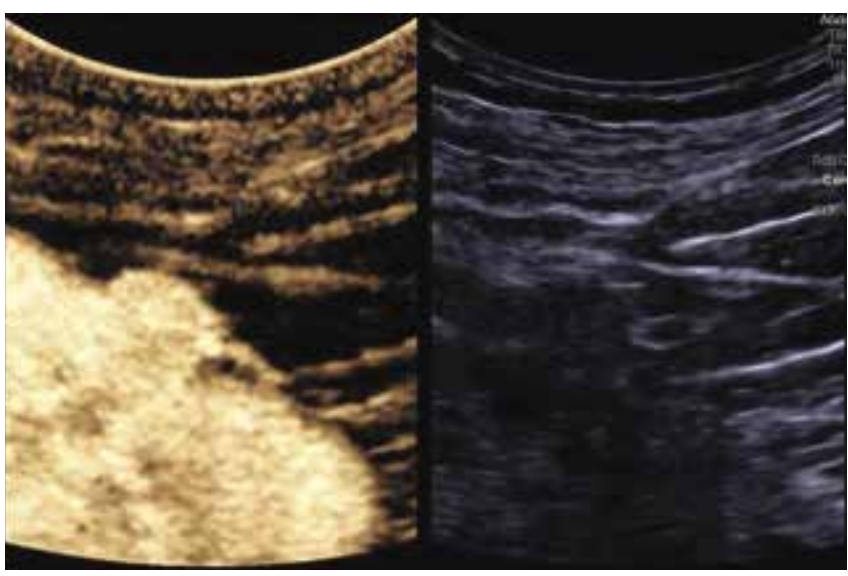

Fig. 5. Intense contrast enhancement of the lesion in the early arterial phase is visible on CEUS images (10 seconds from contrast administration)

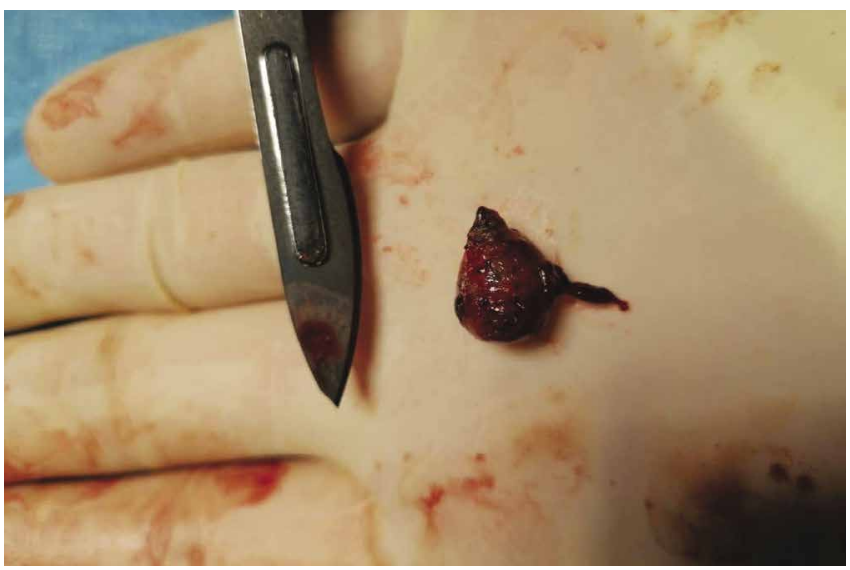

Fig. 7. Intra-operative image depicting the resected tumor; histopathological examination confirmed renal cell carcinoma

between AML and RCC, the enhancement pattern was highly suggestive of malignancy (Fig. 3, Fig. 4, Fig. 5, Fig. 6). The patient underwent laparoscopic organ-sparing resection of the renal tumor. The lesion was removed in its entirety, and the histopathological examination confirmed the diagnosis of RCC (Fig. 7).

\section{Discussion and conclusion}

$80 \%$ of angiomyolipomas are usually detected incidentally during abdominal ultrasound, computed tomography (CT) or magnetic resonance imaging (MRI) ${ }^{(7)}$.

Biopsy is needed for proper diagnosis of AML, however it is very rarely used because of the tumor rupture and bleeding risks.

Ultrasound examination may point towards the diagnosis of AML, however due to its similarity to RCC the following differences should be considered:

- evaluation of external outlines - in AML the borders are smooth and the lesion is well circumscribed;

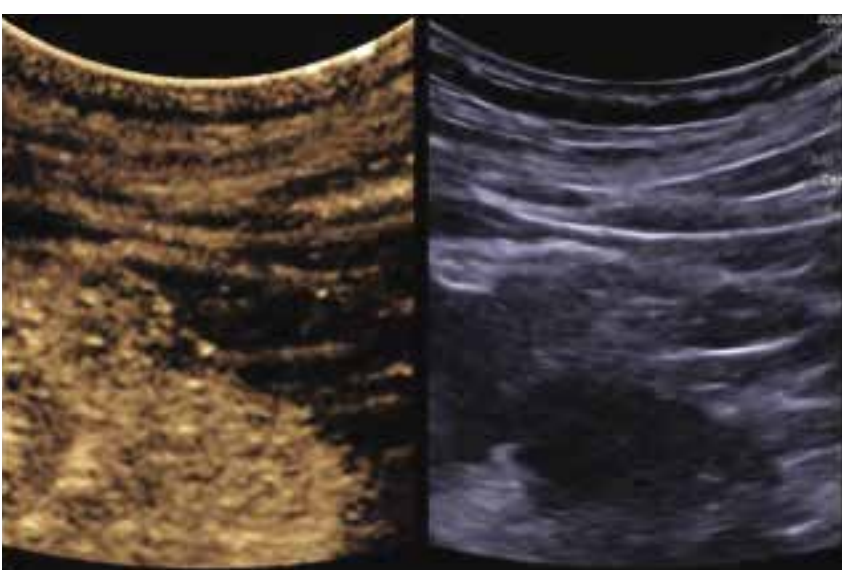

Fig. 6. Relatively earlier contrast washout compared to the surrounding normal renal parenchyma is visible on CEUS images (after 2:50 minutes from contrast administration)

- acoustic shadow - may be seen in some AMLs (21-33\%), but has not been described in renal cell carcinomas;

- AMLs are more often homogeneous lesions. Renal cell carcinoma is more often heterogeneous (65-76\%). Fluid areas are far more common in renal cell carcinoma;

- The presence of an anechoic "halo" around the lesion is more frequently observed in carcinomas than in AMLs;

- Intra-tumoral cysts are found in $73 \%$ of RCCs but are rarely seen in classic $\mathrm{AMLs}^{(8)}$.

CEUS is a fast, low-risk and cost-effective method for the local diagnosis and staging of renal cell carcinoma ${ }^{(9)}$. It can be used to observe the continuous micro- and macrocirculation of a renal mass ${ }^{(10)}$. Benign and malignant kidney lesions can be distinguished since they exhibit a different vascularization pattern to the surrounding healthy renal parenchyma. Furthermore, CEUS is relatively harmless, with no effects on the thyroid and kidney function. It also shows only a low incidence of side effects such as nephrotoxicity ${ }^{(9)}$. CEUS is a valuable real-time diagnostic workup for abdominal emergencies caused by AML or RCC. Besides that, using color-flow Doppler sonography, the blood flow of tumor can be measured, and solid tumor, aneurysm, and pseudoaneurysm can be distinguished. Therefore, despite the lack of accuracy, US has certain clinical value in the diagnosis of AML or $\mathrm{RCC}^{(11)}$.

Other diagnostic procedures such as CT or MRI may help to diagnose AML. The detection of adipose tissue is the fundamental diagnostic criterion in classic AMLs. However, a minority of AMLs lack visually detectable fat on imaging, making them harder to distinguish from renal cell carcinomas $^{(12)}$. In addition, the occurrence of AML and RCC simultaneously may be possible. The presented case shows the differences between various imaging examinations. US revealed the typical picture of AML, which was checked by many by independent ultrasonographers. However, other imaging modalities - CEUS and CT - suggested carcinoma, which was then confirmed histopathologically.

To sum up, AML is mostly detected incidentally during abdominal imaging examinations. Symptoms may not 
occur, therefore a thorough imaging examination plays an important role in the diagnostic work-up of neoplastic lesions in the kidney. Even then, though, the radiological image of the lesion may be misleading, so differential diagnosis is very important for making a proper diagnosis.

\section{References}

1. Arslan B, Gürkan O, Çetin B, Arslan ÖA, Göv T, Yazıcı G et al.: Evaluation of ABO blood groups and blood-based biomarkers as a predictor of growth kinetics of renal angiomyolipoma. Int Urol Nephrol 2018; 50: 2131-2137.

2. Jinzaki M, Silverman SG, Akita H, Nagashima Y, Mikami S, Oya M: Renal angiomyolipoma: a radiological classification and update on recent developments in diagnosis and management. Abdom Imaging 2014; 39: 588-604.

3. Kingswood JC, Belousova E, Benedik MP, Carter T, Cottin V, Curatolo P et al.: Renal angiomyolipoma in patients with tuberous sclerosis complex: findings from the TuberOus SClerosis registry to increase disease Awareness. Nephrol Dial Transplant 2019; 34: 502-508.

4. Park BK: Renal angiomyolipoma based on new classification: how to differentiate it from renal cell carcinoma. AJR Am J Roentgenol 2019; 212: 582-588.

5. Bray F, Ferlay J, Soerjomataram I, Siegel RL, Torre LA, Jemal A: Global cancer statistics 2018: GLOBOCAN estimates of incidence and mortality worldwide for 36 cancers in 185 countries. CA Cancer J Clin 2018; 68: 394-424.

\section{Conflict of interest}

The authors do not report any financial or personal connections with other persons or organizations which might negatively affect the contents of this publication and/or claim authorship rights to this publication

6. Gray RE, Harris GT: Renal cell carcinoma: diagnosis and management. Am Fam Physician 2019; 99: 179-184. Erratum in: Am Fam Physician 2019; 99: 732.

7. Flum, AS, Hamoui, N, Said, MA, Yang XJ, Casalino DD, McGuire BB et al.: Update on the diagnosis and management of renal angiomyolipoma. J Urol 2017; 195: 834-846.

8. Çalışkan S, Gümrükçü G, Özsoy E, Topaktas R, Öztürk Mİ: Renal angiomyolipoma. Rev Assoc Med Bras (1992) 2019; 65: 977-981.

9. Marschner CA, Ruebenthaler J, Schwarze V, Negrão de Figueiredo G, Zhang L, Clevert DA: Comparison of computed tomography (CT), magnetic resonance imaging (MRI) and contrast-enhanced ultrasound (CEUS) in the evaluation of unclear renal lesions. Rofo 2020; 192: 1053-1059.

10. Oh TH, Lee YH, Seo IY: Diagnostic efficacy of contrast-enhanced ultrasound for small renal masses. Korean J Urol 2014; 55: 587-592.

11. Wang C, Li X, Peng L, Gou X, Fan J: An update on recent developments in rupture of renal angiomyolipoma. Medicine (Baltimore) 2018; 97: e0497.

12. Vos N, Oyen R: Renal angiomyolipoma: the good, the bad, and the ugly. J Belg Soc Radiol 2018; 102: 41. 